\title{
Reflets
}

Revue d'intervention sociale et communautaire

\section{La promotion de l'engagement paternel, des archétypes à transformer, une pratique à construire}

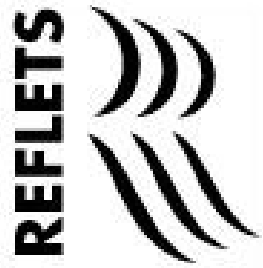

\section{Gilles Forget}

Volume 15, numéro 1, 2009

Paternités : enjeux et perspectives (Première partie)

URI : https://id.erudit.org/iderudit/029588ar

DOI : https://doi.org/10.7202/029588ar

Aller au sommaire du numéro

Éditeur(s)

Reflets : Revue d'intervention sociale et communautaire

ISSN

1203-4576 (imprimé)

1712-8498 (numérique)

Découvrir la revue

Citer cet article

Forget, G. (2009). La promotion de l'engagement paternel, des archétypes à transformer, une pratique à construire. Reflets, 15(1), 79-101.

https://doi.org/10.7202/029588ar

\section{Résumé de l'article}

Le rôle du père à l'égard du développement, de la santé et du bien-être de l'enfant et la place qu'on lui accorde dans les services sont une préoccupation croissante des communautés scientifique et politique. Des milieux de pratiques se sont aussi intéressés au rôle du père dans un contexte où de nombreux changements sont survenus au sein des familles. Les attentes sociales envers les pères sont teintées par des archétypes qui balisent les rôles des parents de même que les services que l'on prévoit à leur endroit. Ces archétypes voient toujours l'apport particulier des mères au développement de l'enfant et relèguent le père au rôle de pourvoyeur. Interroger les intervenants sur la place des pères dans leurs interventions les amène à revoir leurs perceptions sur la contribution de ces derniers au développement de l'enfant, de même que leurs attitudes et leurs approches auprès d'eux. Le transfert des connaissances issu des travaux de l'équipe ProsPère, associée au Groupe de recherche sur la victimisation des enfants, s'est fait de différentes façons. Pour rejoindre les intervenants, des membres de l'équipe ont élaboré, validé et déployé au Québec, au Canada et en France une formation. Une réflexion à partir de notre expérience en tant que chercheur et formateur servira à illustrer certains principes qui favorisent une meilleure prise en compte des besoins des pères dans les services de même que certaines barrières à ce sujet. Cette réflexion servira aussi à proposer des balises pour construire de nouvelles pratiques favorisant l'engagement paternel.
Tous droits réservés (C) Reflets : revue d'intervention sociale et communautaire, 2009
Ce document est protégé par la loi sur le droit d'auteur. L'utilisation des services d'Érudit (y compris la reproduction) est assujettie à sa politique d'utilisation que vous pouvez consulter en ligne. 


\section{La promotion de l'engagement paternel, des archétypes à transformer, une pratique à construire}

\section{Gilles Forget}

Conseiller en promotion de la santé, Direction de santé publique de l'Agence de la santé et des services sociaux de Montréal

L'objectif général du Groupe de recherche et d'action sur la victimisation des enfants - Alliance de recherche pour le développement des enfants dans leur communauté (GraveArdec $)^{2}$ est de contribuer, par l'acquisition de connaissances, à l'amélioration des contextes et des services aptes à prévenir la victimisation des enfants et à en atténuer les conséquences. La première demande de financement de cette équipe présente l'engagement paternel comme une stratégie préventive de la victimisation des enfants ${ }^{3}$. Les raisons invoquées tiennent au rôle du père dans le développement des enfants, qu'il soit positif ou négatif, et à la nécessité de développer des pratiques innovantes dans un domaine jusqu'alors peu exploré. Pour atteindre cet objectif, des membres du Grave-Ardec, chercheurs universitaires, chercheurs institutionnels et intervenants, constituent l'équipe qu'ils nommeront ProsPère. Leur programmation de recherche se développe selon trois axes, une réflexion sur le sens et les déterminants de la paternité, une recherche théorique sur l'attachement et l'évaluation de pratiques innovantes. Après quelques années de travail, le transfert de ces connaissances avec les milieux de pratique s'est réalisé notamment à l'aide d'une formation à l'intention des intervenants des différents réseaux. 
La formation Pères en mouvement, pratiques en changement présente les résultats des recherches-actions de l'équipe et ceux d'autres recherches scientifiques et mise sur la pédagogie des adultes pour animer la réflexion des participants autour du rôle du père, de sa place dans les services, de l'impact du processus migratoire et du développement d'une pratique tant individuelle que collective de promotion de l'engagement paternel.

\section{Une vision de l'engagement paternel}

«Ces recherches... concourent à voir une contribution spécifique du père à l'égard de son enfant qualifiée d'ouverture au monde qui permet à l'enfant d'avoir confiance en ses propres capacités pour faire face aux dangers et à l'étrangeté de l'environnement. Ces nouvelles réflexions concourent à accorder plus d'importance au rôle du père dans le développement de l'enfant."
Pour amorcer les travaux, l'équipe a fait le point sur les connaissances portant sur l'engagement paternel et recherché un consensus sur une définition de même que sur une approche de promotion de l'engagement paternel. Au début des années 90, le monde scientifique s'intéresse de plus en plus à la paternité et à son effet sur le développement des enfants. Les approches théoriques prédominantes à cette époque sont celle de l'attachement, celle du développement et de l'adaptation sociale de l'enfant et celle d'une approche différenciée selon le genre. Le modèle théorique de l'attachement se développe pour mieux comprendre les problèmes émotionnels chez les enfants. L'attachement est alors conçu comme faisant partie des besoins primaires : de même qu'il doit s'alimenter pour grandir, le bébé doit aussi, pour se développer et explorer le monde, pouvoir trouver sécurité et réconfort par un lien privilégié avec l'adulte (Ainsworth, 1972; Bowlby, 1969). Au fil des années, ce modèle théorique a soulevé certaines critiques notamment, celle de donner un rôle central à la mère, et de cantonner ainsi la femme dans un schéma très conformiste. Les recherches ultérieures viennent compléter cette théorie. Ainsi, un des chercheurs de l'équipe s'est particulièrement intéressé à la dimension "exploration » (Paquette 2004a, 2004b) de cette théorie de l'attachement. Ces recherches, combinées à celles de chercheurs français (Le Camus, 2004) concourent à voir une contribution spécifique du père à l'égard de son enfant qualifiée d'ouverture au monde qui permet à l'enfant d'avoir 
confiance en ses propres capacités pour faire face aux dangers et à l'étrangeté de l'environnement. Ces nouvelles réflexions concourent à accorder plus d'importance au rôle du père dans le développement de l'enfant.

Les recherches américaines sur le développement et l'adaptation sociale de l'enfant ont donné lieu, à la fin des années 80 , à une définition de cet engagement (Lamb, 1987) qui inclut trois composantes principales, soit les interactions directes du parent avec l'enfant, la disponibilité ou l'accessibilité et la responsabilité parentale.

Cette définition découle des résultats de recherches qui soulignent l'influence bénéfique de l'engagement du père sur le développement cognitif, émotif et social de l'enfant et réduit la délinquance, la prise de substance et la fréquence de comportements internalisés, dépression, et externalisés, les manifestations agressives (Allen et Daly, 2002). Se basant sur cette définition, des recherches ont contribué à mesurer l'engagement paternel (Lamb, 1987). Ces mesures se limitent aux comportements des pères envers l'enfant, omettant d'autres facettes de leur engagement. Ces travaux ont contribué à reconnaître, au fil des ans, l'engagement accru des pères auprès de leurs enfants (Pleck et Masciadrelli, 2004). Ils ont également incité de nombreux chercheurs à investiguer les déterminants associés à cet engagement afin d'identifier les caractéristiques personnelles, familiales et sociales qui amenaient certains pères à être plus engagés que d'autres. Cette définition s'inscrit enfin dans la tradition américaine qui cherche à rendre les pères plus responsables, les incitant surtout à verser régulièrement leur pension alimentaire. Par ailleurs, le rôle de pourvoyeur est considéré incomplet. Le cash and care father constitue une perception de la paternité qui fait suite aux transformations des rôles des pères et mères qui sont dorénavant tous les deux présents sur le marché du travail et responsables de la prestation des soins aux enfants (Hobson et Morgan, 2002). Dans cette foulée, l'approche centrée sur le développement de l'enfant a aussi fait apparâtre aux États-Unis une vision plus globale de l'engagement paternel.Ainsi, plusieurs études ont en commun l'adoption de cadres d'analyse inspirés du modèle écologique (Belsky, 1984; Doherty, Kouneski 
"La définition retenue repose sur le principe qu'être parent est un rôle en continu qui s'amorce dès la décision d'avoir un enfant. Elle prend en compte les nouvelles réalités familiales en insistant sur le fait que le rôle de père peut être joué par d'autres hommes que le père biologique et que ce rôle se réalise tout autant en présence de l'enfant qu'à distance. " et Erickson, 1998; Lamb, Pleck et Levine, 1985) reconnaissant que le niveau d'engagement paternel résulte de l'interaction dynamique de facteurs relevant à la fois de caractéristiques du père, des enfants, de la mère et du contexte social.

Une autre façon d'entrevoir la place du père dans le développement de l'enfant est ce que d'aucuns nomment l'approche essentialiste. Elle prend appui sur l'augmentation importante des ruptures dans les couples pour interroger l'absence du père, le Fatherless America (Blankenhorn, 1995). Selon cette approche, un des éléments manquants à l'analyse des problèmes sociaux est l'apport spécifique des pères. Il lie l'absence des pères aux nombreux problèmes sociaux (décrochage scolaire, violence agie et subie) et de santé qui affligent les enfants et les adolescents. Cette analyse selon le genre, amène l'auteur à conclure à une contribution unique du père au développement de l'enfant. Une autre conception de la paternité à l'aide d'une analyse selon le genre est celle de la générativité (Hawkins et Dollahite, 1997) qui insiste sur la filiation entre le père, l'homme, et ses enfants et les aspects bénéfiques de l'engagement paternel pour la santé et le mieux-être du père lui-même. Ce concept souligne également que, même en contexte de précarité et de vulnérabilité, les pères peuvent contribuer au bien-être de leur enfant.

À la lumière de ces courants idéologiques et de ces recherches, l'équipe a adopté sa propre définition de l'engagement paternel : "L'engagement paternel est la participation et la préoccupation continue du père biologique ou substitut envers le développement physique, psychologique et social de son enfant». Cette définition se base sur sept dimensions, le père pourvoyeur, le père responsable, le père en interaction, le père qui prend soin, le père affectueux, le père évocateur et le père citoyen. Ce consensus d'une équipe composée d'hommes et de femmes n'est pas venu sans débats houleux, à l'image du débat social qui entoure la transformation des rôles sociaux et celui de père et de mère. Enfin, ce consensus s'est surtout formé grâce au temps accordé aux discussions sur chacune des perspectives et au fait qu'il tient compte des nouvelles configurations familiales des mères et des pères. La définition retenue repose sur le principe qu'être parent est un rôle en 
continu qui s'amorce dès la décision d'avoir un enfant. Elle prend en compte les nouvelles réalités familiales en insistant sur le fait que le rôle de père peut être joué par d'autres hommes que le père biologique et que ce rôle se réalise tout autant en présence de l'enfant qu'à distance. De plus, afin d'englober l'ensemble des facettes associées à ce rôle, elle s'étend bien au-delà de celle de la responsabilité du père envers son enfant. Elle insiste sur le fait que le rôle de père se manifeste autant dans la sphère domestique que dans la sphère publique. Elle reconnaît ses qualités d'antan, le père pourvoyeur, et sa façon unique d'accompagner l'enfant dans son ouverture au monde. Cette façon d'envisager le rôle et la place du père à l'égard du développement de l'enfant propose un nouvel archétype de la paternité qui est multidimensionnel. Cette définition propose à sa façon une nouvelle attente de la société à l'égard du rôle et de la place du père face à son enfant (Pitt, 2001).

\section{Des recherches-actions et l'analyse des services}

Comment alors faire la promotion de l'engagement des pères? Quelles sont les pratiques à mettre en place, pratiques qui tiennent compte de cette réalité et suscitent, renforcent et maintiennent l'engagement paternel. L'équipe s'est appuyée sur deux leviers pour faciliter l'élaboration et le transfert de connaissances. Elle a réalisé ses propres recherches-actions avec des communautés, des entreprises et des organismes communautaires. Elle a aussi réalisé de nombreuses études sur les pratiques en cours et suivi leur évolution.

La première expérience fut celle de l'implantation d'un projet d'action communautaire sur l'engagement paternel (Turcotte, Desjardins et Ouellet, 2001). En s'appuyant sur l'approche écologique du développement de la paternité (Bronfenbrenner, 1979), les interventions mobilisent plusieurs partenaires de divers secteurs et mettent en place des interventions variées et de multiples stratégies pour influencer les différents facteurs liés à 
l'engagement paternel présents dans les communautés. La première recherche-action se réalise grâce à la participation et au soutien des partenaires du réseau de la santé, des services sociaux et des acteurs communautaires de deux sites de la région montréalaise qualifiés de vulnérables de par la situation socio-économique des familles et le nombre élevé d'enfants victimes de différents types d'abus ou de négligence. Cette première expérience s'est déroulée de 1995 à 2002 et a généré plusieurs activités dans ces deux sites et l'expérimentation de moyens novateurs de transfert des connaissances auprès des décideurs, de la communauté scientifique, des intervenants et de la population (Dubeau, 2007). À l'image de la démarche de l'équipe autour de la définition de l'engagement paternel, l'expérience vécue par les partenaires des deux sites montre également qu'un laps de temps important est nécessaire pour faire face au défi de se doter d'une conception commune de la place et du rôle du père. Les discussions avec les partenaires soulèvent l'inscription de la promotion de l'engagement paternel dans le débat social sur les rapports de genre, la nécessité de discuter des rôles sociaux et l'importance de se questionner sur ses propres valeurs. La recherche a maintenu un lien continu avec les terrains et organisé sur les lieux et avec les acteurs plusieurs réunions scientifiques ${ }^{4}$ qui ont facilité l'échange de connaissances entre les partenaires et leur mobilisation pour implanter ce projet. Les deux communautés ont proposé des plans d'action annuels et réalisé de nombreuses interventions auprès des pères, des mères et des enfants. Ils ont aussi diffusé les résultats des enquêtes menées de diverses façons auprès des pères et mères, dont l'exposition itinérante Dessine-moi un papa et les Accroches-pères.

Les recherches qui suivent cette première expérience explorent de nouvelles situations pour promouvoir l'engagement paternel et évaluent de nouvelles pratiques de soutien aux pères. La recherche-action Métiers de pères s'est déroulée dans des entreprises d'insertion sociale de la région montréalaise pour mieux connaître les réalités des jeunes pères et les façons d'associer leur paternité dans la démarche d'insertion qu'ils sont à réaliser (Devault, 2005). Ces entreprises représentent un outil que s'est donné le Québec pour contrer le phénomène de l'exclusion sociale des jeunes. Leur 
"Cette rechercheaction permet de mieux comprendre les trajectoires personnelles qui influencent l'expérience de la paternité vécue par ces jeunes hommes. Elle souligne aussi l'importance de la paternité pour l'insertion de ces pères et pousse l'équipe à tenter de mieux rejoindre les pères vulnérables." mission principale est de former les jeunes à un métier (ébénisterie, pliage du métal, entretien ménager...) et de les soutenir dans leur recherche d'emploi. Les entreprises d'insertion représentent pour plusieurs raisons un formidable potentiel d'intervention auprès de jeunes pères en situation de précarité. Elles rejoignent une jeune clientèle (15-25 ans) majoritairement masculine. Elles offrent des services à une population vulnérable économiquement qui ne fréquente pas nécessairement d'autres services, par exemple les Centres de services de santé et de services sociaux (CSSS) ou les Maisons de la famille. Elles rejoignent des personnes qui ont de jeunes enfants ou qui sont sur le point de devenir parents. Enfin, les entreprises d'insertion offrent un véritable milieu de vie aux jeunes adultes. Cette recherche-action permet de mieux comprendre les trajectoires personnelles qui influencent l'expérience de la paternité vécue par ces jeunes hommes. Elle souligne aussi l'importance de la paternité pour l'insertion de ces pères et pousse l'équipe à tenter de mieux rejoindre les pères vulnérables.

La troisième recherche-action, dont les résultats sont à être diffusés $^{6}$, se déroule dans la région montréalaise en partenariat avec les acteurs de quatre quartiers. Mis en place depuis l'été 2005, Relais-Pères soutient les pères d'enfants âgés de 0 à 5 ans, résidant ou non avec eux et répondant aux critères de vulnérabilité du Programme des services intégrés en périnatalité et en petite enfance (SIPPE), c'est-à-dire, avoir moins de 20 ans, ne pas avoir complété ses études secondaires, avoir un revenu se situant sous le seuil de faible revenu tel que défini par Statistique Canada ou cumuler plusieurs facteurs de vulnérabilité liés à un parcours migratoire récent. L'accompagnement offert par des intervenants de milieu permet à ces pères d'inclure le développement de l'enfant dans un projet porteur de réussite et de favoriser leur insertion sociale. L'évaluation de cette expérience indique que ce type d'intervention rejoint la clientèle ciblée et favorise le continuum des services auprès de la population vulnérable par le relais entre les services et les ressources dans les quartiers où se sont déroulés les projets. 
En somme, tout au long de ces recherches, l'équipe de ProsPère s'est non seulement inspirée des plus récentes recherches scientifiques sur la paternité, sur le développement des enfants et sur les pratiques prometteuses à mettre en place, elle s'est aussi inspirée des besoins de la population et des savoirs d'expérience de tous ses collaborateurs. Elle a finalement réalisé plusieurs études sur les pratiques de soutien aux pères.

\section{L'exemple d'autres initiatives et le suivi de leur évolution}

Tout au long du développement des recherches, des membres de l'équipe de ProsPères ont réalisé ou collaboré à des enquêtes auprès d'organismes montréalais, québécois et canadiens afin de préciser les pratiques en cours pour soutenir les pères. En plus de décrire ces pratiques, ces enquêtes permettent de préciser leurs conditions de succès. L'analyse de milieu (Forget, 1995) réalisée lors de la première recherche a permis de préciser les besoins des pères et des mères, les caractéristiques des ressources de la communauté et la place qu'elles font aux pères, les barrières et les opportunités pour l'implantation d'un projet de promotion de la santé (Rissel et Bracht, 1999). D'autres études (Arama et Bouchard, 1996; Arama, 1997) ont été réalisées auprès de gestionnaires et d'intervenants des réseaux institutionnel et communautaire des différentes régions du Québec. Une première enquête canadienne réalisée par des membres de l'équipe ProsPère et des partenaires ontariens, Sur le terrain des pères (Bolté et collab., 2002), fait l'inventaire des pratiques et souligne, à l'aide d'une étude en profondeur de quinze de celles-ci, dont celles réalisées dans les deux communautés associées à la recherche-action de ProsPère, les conditions qui entourent ces pratiques et en assurent le succès. S'appuyant sur ces résultats, une seconde enquête réalisée auprès d'un plus grand nombre d'initiatives des différentes régions canadiennes (Forget et collab., 2005) précise certains obstacles auxquels ces organismes font face pour améliorer le soutien aux pères. Cette enquête a aussi 
permis le suivi des pratiques de 55 organismes ayant participé aux deux enquêtes $(1999,2003)$. La comparaison entre les réponses des organismes qui ont participé aux deux enquêtes et ceux qui n'ont répondu qu'à la plus récente suggère que la longévité des pratiques influence l'offre de services, les programmes plus anciens étant proportionnellement plus nombreux à offrir une gamme d'interventions aux pères. De plus, ceux qui offrent des services aux pères depuis plus longtemps rejoignent davantage les pères en situation de vulnérabilité, c'est-à-dire, les plus jeunes, les plus pauvres et les pères séparés. Enfin, ceux dont les services sont offerts depuis plus longtemps n'identifient plus les heures d'ouverture et la formation de leur personnel comme des obstacles au soutien aux pères. L'analyse de l'évolution des pratiques indique clairement que celles propres aux pères, appuyées par un financement récurrent avec des intervenants formés et dédiés, sont les pratiques qui atteignent le mieux l'objectif de susciter, de renforcer et de maintenir l'engagement des pères auprès de leurs enfants.

\section{Partager les connaissances, la formation sur le terrain}

Comme le prévoit la recherche-action, l'équipe a partagé les connaissances découlant de ses études avec les intervenants, constatant le levier extraordinaire que représentent leurs activités quotidiennes pour la promotion de l'engagement paternel. Deux des membres de l'équipe élaborent alors la formation Pères en mouvement, pratiques en changement diffusée à Montréal et dans différentes régions du Québec (Ouellet, 2001). Les versions anglophone et internationale de la formation ont permis de rejoindre des intervenants de provinces canadiennes et de certaines régions de France (Forget, 2002; Forget, 2006). Cette formation vise à apporter une meilleure compréhension de l'importance de l'engagement paternel, à développer des services sympathiques aux pères et à disposer de pistes d'interventions concrètes, individuelles 
"Tout au long de ces formations, les formateurs ont vite constaté que le sujet de la promotion de l'engagement paternel suscitait énormément d'émotions et de débats qui ne sont pas sans rappeler ceux vécus au sein de l'équipe. » et collectives, autour de la promotion de l'engagement paternel. Ces formations ont permis aux chercheurs associés à ProsPère, devenus formateurs, de rencontrer des centaines d'intervenants de différents secteurs dans des contextes variés. Bien qu'un effort ait été fait pour assurer une représentativité égale d'intervenants et d'intervenantes, les groupes ont surtout été composés d'intervenantes, reflétant la réalité du réseau de la santé et des services sociaux venant en aide aux familles. Tout au long de ces formations, les formateurs ont vite constaté que le sujet de la promotion de l'engagement paternel suscitait énormément d'émotions et de débats qui ne sont pas sans rappeler ceux vécus au sein de l'équipe.Trois enjeux revenaient à coup sûr dans chacune des formations. Pourquoi faire la promotion de l'engagement paternel ou aider des pères qui sont absents, violents ou irresponsables? Dans la majorité des groupes, les intervenantes préoccupées par les risques pour l'enfant de comportements paternels abusifs s'interrogent sur l'intérêt de favoriser l'engagement des pères. Comment les rejoindre et les accompagner? Les participants ayant déjà tenté, sans succès, de rejoindre les pères posent fréquemment cette question et manifestent aussi leurs préoccupations à l'égard d'actions qui sont susceptibles de soutenir les pères. Comment aider les pères et inscrire ces actions dans un quotidien déjà submergé par les demandes des mères? La réalité de ressources sous financées, suffisant à peine à assurer une action de qualité auprès des mères à cause d'une demande trop grande fait en sorte que plusieurs participants voient difficilement comment ils peuvent aussi rejoindre et soutenir les pères. Néanmoins, un premier bilan (Ouellet, 2003) de cette formation indique sa capacité à susciter le développement de nouvelles pratiques auprès des pères. Ainsi, après chacune des formations, des participants indiquent comment ils ont mis en pratique le contenu proposé, soit par des changements sur le plan de l'environnement physique de leurs services par la mise en place d'affiches illustrant des pères en interaction avec leurs enfants, soit dans leurs pratiques individuelles en s'informant auprès des mères de la situation du père, soit par la mise en place d'activités familiales où sont invités nommément les pères. Notamment, plus d'intervenants sollicitent les pères; la 
communication avec ces derniers s'est bonifiée et la promotion des activités est mieux adaptée. Une évaluation subséquente (Venet, 2006) révèle en outre que les changements de pratique observés chez les intervenants se maintiennent dans le temps.Ainsi, les changements notés par les intervenants lors d'une première entrevue à la suite de la formation, une promotion des activités mieux adaptées ou des changements apportés aux horaires des activités sont toujours en place lors de la seconde entrevue réalisée plus de six mois après la première.

\section{Des archétypes à transformer}

«Euvrer à prévenir la victimisation des enfants par la promotion de l'engagement paternel était à la source des travaux de l'équipe ProsPère, un sujet relativement nouveau ou, à tout le moins, qui n'avait pas fait l'objet de plusieurs expérimentations. "
CEuvrer à prévenir la victimisation des enfants par la promotion de l'engagement paternel était à la source des travaux de l'équipe ProsPère, un sujet relativement nouveau ou, à tout le moins, qui n'avait pas fait l'objet de plusieurs expérimentations. Les discussions entre membres, avec les partenaires, avec les intervenants et avec des pères et des mères ont tôt fait de révéler que le sujet de la paternité nous renvoie tous à ces archétypes du père, de la mère, du rôle de chacun et de l'occupation de l'espace domestique et public par chacun des genres. Le père a été longtemps détenteur d'un pouvoir quasi absolu sur les mères et les enfants. L'évolution des sociétés occidentales remet en question cette autorité et, actuellement, la charte des droits et libertés, les lois et les réglementations ne permettent plus de distinction entre l'autorité et les responsabilités assumées par les pères et les mères. Ainsi, l'archétype du père autoritaire diminue dans la représentation contemporaine de la paternité. Si le père n'est plus autorité, il n'est plus aussi le seul pourvoyeur. Même s'il reste inférieur à celui des hommes, le revenu des femmes a augmenté et des secteurs importants de l'économie sont féminins, particulièrement dans tout ce qui entoure l'enfant, la santé, l'éducation, les services de garde à l'enfance ou les organismes communautaires famille. La conciliation travail-famille est devenue un défi pour nombre de ménages. La famille où chacun des parents exerce des tâches 
spécifiques cède donc la place à une nouvelle représentation de la parentalité qui insiste sur la complémentarité, les discussions et les négociations comme fondements du rôle de parent. Enfin, la paternité présente de multiples configurations, à l'image des familles de notre société.

\section{La paternité, une expérience universelle et personnelle}

Tout au long des formations, où qu'elles se soient déroulées, les participants n'ont pu aborder le sujet de la paternité sans se sentir personnellement interpellés. Le sujet de la paternité n'est pas un sujet neutre. Individuellement, il nous renvoie à notre propre père. Collectivement, il évoque les relations entre les genres et la recherche d'égalité et d'équité. Pour envisager positivement le rôle et la place du père et vouloir accompagner les pères qui fréquentent les services, il faut faire la paix avec ces images et voir les effets positifs de la paternité pour l'homme lui-même, le couple et l'enfant.

\section{Intervenir au masculin}

Lors des recherches-actions, l'importance d'intervenir au masculin est régulièrement mentionnée. On observe des manières différentes qu'ont les pères d'interagir avec l'enfant, des effets spécifiques sur le développement des enfants ou des conditions d'exercice de la parentalité qui diffèrent selon le genre. Ces analyses ont suscité plusieurs débats entre les chercheurs du groupe, certains faisant la promotion d'une approche différenciée selon le genre, d'autres liant ces façons de faire à des contextes sociaux différents. Ces débats trouvent écho dans les échanges entre les formateurs et les participants. L'échange entre ce groupe d'hommes et de femmes soulève aussi la nature des relations qu'ont les intervenants avec les familles. La question demeure ouverte. Mais l'analyse différenciée 
selon le genre a l'avantage de soulever, que ce soit pour les femmes comme pour les hommes, la variété des situations, des contextes, des conditions d'exercice de la paternité qui reflètent la situation sociale actuelle. Elles soulèvent aussi pour les intervenants les efforts à faire pour répondre aux besoins particuliers des hommes.

L'analyse des services offerts au père nous amène à réfléchir à la demande d'aide des hommes. Non seulement demandent-ils à leur façon, mais ils sont aussi sensibles au genre de l'intervenant. Les hommes et les femmes ont des façons particulières d'entrer en relation, de communiquer mutuellement, d'interagir. Mais cette présence d'intervenants masculins soulève de nouveaux enjeux. La présence d'un intervenant masculin dans un monde d'intervenantes n'est pas sans bousculer les idées dont se font les intervenantes d'un accompagnement personnalisé. Comme le fait ressortir l'approche de l'intervenant communautaire de RelaisPères, l'intervention d'un intervenant masculin fait de concert avec une intervenante amène celle-ci à revoir sa propre vision des pères et à bonifier ses interventions auprès des familles. Elle vient finalement faciliter l'intervention auprès des deux parents à la fois, en offrant deux modèles de genre. Offrir des services aux deux parents simultanément constitue une piste prometteuse d'intervention favorisant l'engagement des pères auprès de leur enfant. En effet, de cette façon, les besoins des deux parents sont pris en considération dans l'organisation de la vie de la famille et concernant les rôles que chacun joue en son sein.

\section{Des pratiques à construire}

L'échange entre les savoirs théoriques et pratiques a amené l'équipe ProsPère à établir certains principes d'intervention. D'abord, de façon générale, il est crucial d'intervenir globalement sur le plus d'aspects susceptibles d'influencer l'engagement des pères. Cette considération implique une collaboration étroite entre différents services, acteurs locaux et intervenants. De façon plus particulière, certains principes s'avèrent très éclairants pour guider la réflexion 
"Bien que nos sociétés mettent de l'avant l'importance de la présence des deux parents pour favoriser la santé et le bien-être des enfants pour leur avenir, plusieurs des programmes de soutien aux familles visent uniquement les mères à toutes les étapes de leur vie. » sur la paternité et les actions qui visent à favoriser l'engagement des pères envers leur enfant.

\section{Valoriser d'abord le rôle et la place du père}

Bien que nos sociétés mettent de l'avant l'importance de la présence des deux parents pour favoriser la santé et le bien-être des enfants pour leur avenir, plusieurs des programmes de soutien aux familles visent uniquement les mères à toutes les étapes de leur vie. Sans revenir sur l'évolution de nos sociétés des dernières décennies, et particulièrement sur l'évolution des configurations familiales, la hausse vertigineuse des unions libres, la décroissance des mariages, les ruptures familiales, le taux élevé de pauvreté chez les enfants ou la diversité des familles, le rôle et la place du père dans les familles demeurent négligés malgré les avancées des plus récentes politiques familiales (congé parental). Cette situation est mentionnée explicitement dans la récente politique de périnatalité du Québec :

Il ne faut pas négliger le rôle du père. Le père d'aujourd'hui joue en effet un rôle différent de celui d'hier. Certes il n'y a pas de modèle unique: on constate plutôt une riche diversité de modèles. Les services de périnatalité doivent s'adapter à cette diversité et mieux intégrer les pères aux interventions et montrer toute l'ouverture nécessaire à leur égard. Bref, l'implication du père au cours de la période périnatale doit être reconnue, favorisée et soutenue. (Québec, 2008, p. 4)

C'est ainsi que les milieux où se sont implantées les recherchesactions ont tous intégré à leurs premières interventions des activités de sensibilisation des parents, des intervenants et de la population à l'importance du rôle et de la place du père. Ce faisant, ils valorisent les pères et leur montrent que leurs services sont soucieux de leurs besoins. 


\section{Aller là où sont les pères}

Plusieurs organismes-familles interrogés sur leurs pratiques auprès des pères (Forget, 2005) répondent qu'un de leurs principaux problèmes pour développer des activités, services, programmes ou ressources pour les pères sont les pères eux-mêmes. Bien qu'ils soient les bienvenus aux activités de l'organisme, ils ne s'y présentent pas. Les expériences décrites ont toutes en commun cet effort d'aller là où sont les pères. Dans les communautés, des efforts particuliers de démarchage dans les endroits publics ont donné lieu à la mise en place de groupes de pères dont certains ont participé aux activités de l'organisme pendant plusieurs années. Par exemple, l'alliance avec les entreprises d'insertion misait sur le fait que celles-ci regroupaient de nombreux jeunes hommes. Les intervenants se sont vite aperçus que plusieurs d'entre eux étaient pères. Enfin, cette approche que l'on pourrait qualifier de proximité, utilisée dans le dernier projet amène les intervenants à prendre du temps pour se faire connaître des services et ressources du quartier et à rejoindre les pères dans les lieux qu'ils fréquentent au quotidien.

\section{Mettre en relation le père avec son enfant}

Rejoindre les pères constitue une façon de leur apporter du soutien. Par ailleurs, les mettre en action avec leur enfant constitue une voie privilégiée de favoriser leur engagement et leurs forces. Par exemple, les pères semblent jouer un rôle particulier dans l'ouverture au monde reliée tout particulièrement à l'autonomie et à la gestion de la prise de risques au cours de l'exploration des environnements physique et social (Paquette, 2004b). Cette particularité paternelle favorise le développement des habiletés physiques et sociales, de l'affirmation de soi, la gestion de la colère dans les rapports sociaux avec les pairs et la réussite scolaire. Ainsi, aider les pères à reconnaître leur contribution constitue des interventions directes auprès d'eux pouvant apporter des résultats tangibles et susceptibles de les encourager. En multipliant les contextes d'apprentissage des enfants, les différences entre le père et la mère constituent une plus grande richesse pour les enfants 
que l'homogénéisation des pratiques parentales (Paquette, 2004b). Lorsqu'il est question d'activités favorisant l'engagement des pères, les expériences nous montrent l'énorme potentiel d'activités pères-enfants. Elles leur offrent la possibilité d'interagir avec leurs enfants, ce que certains n'ont pas l'occasion d'expérimenter, dans un espace sécuritaire où jouer avec l'enfant, une façon de sortir de leur isolement et un lieu pour échanger avec d'autres pères.

\section{Adopter une approche familiale}

La paternité s'exerce en relation avec l'enfant et l'autre parent. Le soutien accordé aux pères ne doit pas oublier l'action à faire auprès des mères pour que celles-ci s'adaptent aux transformations des rapports sociaux et à l'inclusion du père dans l'espace domestique. Bien qu'elles visent et rejoignent les pères, la majorité des activités ne négligent aucunement le soutien à accorder aux mères. Cette approche familiale accroît ainsi le potentiel d'impact sur le développement, la santé et le bien-être des enfants.

\section{Appuyer la coparentalité}

La " coparentalité " définit les relations entre conjoints ou ex-conjoints qui ont trait à la parentalité et à l'éducation des enfants. Comme le mentionnent certains auteurs (Margolin, Gordis et John, 2001), c'est à travers la relation coparentale que les parents négocient leurs rôles, responsabilités et contributions respectifs à l'égard de leurs enfants. En plus de comporter de nombreux bénéfices pour les enfants, une coparentalité de qualité s'accompagne habituellement d'une relation conjugale plus satisfaisante, de croyances parentales moins contraignantes et d'un style parental plus adéquat (Gagnon, 2009). Afin de traduire ces connaissances et les partager avec les jeunes pères, la rechercheaction Métiers de pères a produit un feuillet distribué aux jeunes fréquentant les entreprises d'insertion. La coparentalité y est décrite de la façon suivante :

"Pour réaliser son rôle de père et de mère, il faut développer le travail d'équipe en: 
- construisant une relation de collaboration pour protéger les enfants des conflits parentaux

- s'assurant que chaque parent fasse une place à l'autre

- installant un climat de respect, de sécurité et de stabilité pour les enfants, et ce, malgré les circonstances de la vie

- en se consultant sur les grandes questions qui concernent l'orientation, l'éducation et la santé de leurs enfants

- en contribuant selon leurs moyens respectifs aux coûts associés au développement et au bien-être des enfants. "

Susciter l'engagement des pères dans les communautés, mais aussi à l'intérieur des services

Créer des environnements sympathiques aux pères a toujours été un objectif des actions de ProsPère. L'analyse des services des communautés (Arama, 1997; Bolté et collab., 2002) démontre que plusieurs d'entre eux ne sont pas aussi accueillants pour les pères qu'ils pourraient l'être. Ainsi, les communautés ont produit différents outils de communication pour ces derniers, affiches, feuillets, expositions, brochures, et les ont diffusés dans les différentes ressources afin de faire sentir aux pères qui fréquentent ces services qu'on s'intéresse à eux. Dans les entreprises d'insertion, cette action s'est traduite notamment, par la mise en place d'un

"Comme le révèle la dernière expérience, les pratiques efficaces sont celles qui couvrent un large spectre de besoins qui ne peuvent être répondus par un seul intervenant ou un même organisme. Le relais aux autres services de la communauté devient alors une composante essentielle de l'action." babillard où les pères pouvaient mettre des photos d'eux et de leurs enfants. Elles ont aussi installé dans leurs locaux des affiches sur l'engagement des pères. Enfin, les travailleurs de milieu influencent aussi l'accueil des pères dans les organismes des milieux en les y accompagnant et participant aux activités des organismes. Pour ceux qui veulent rendre plus sympathique leur environnement aux pères, il ne faut pas oublier non plus la façon de les accueillir au téléphone ou en personne, l'établissement d'une relation de confiance, d'ouverture et de respect.

\section{Favoriser les relais entre les ressources}

Comme le révèle la dernière expérience, les pratiques efficaces sont celles qui couvrent un large spectre de besoins qui ne peuvent être répondus par un seul intervenant ou un même organisme. Le relais aux autres services de la communauté devient alors une 
composante essentielle de l'action. Pour ce faire, l'intervenant doit d'abord connaitre les ressources du milieu, et s'en faire reconnaitre, afin de faciliter les relais entre les différents services qui facilitent l'engagement des pères envers leurs enfants. Soutenir les pères implique aussi un élargissement de l'action. L'engagement des pères se fait de façons variées et ne concerne pas uniquement les interactions avec l'enfant. Son insertion au monde de l'emploi, les négociations avec les réseaux des services sociaux et juridiques, le logement ou encore le soutien à l'éducation sont des aspects importants de ses trajectoires personnelle, sociale et parentale. Donc, la concertation entre les organismes pour favoriser l'utilisation de leurs services par les pères a son importance. Il en va de même pour les intervenants d'accompagner les pères d'une ressource à une autre pour favoriser une continuité.

\section{S'assurer d'un financement constant et croissant}

L'équipe de recherche a bénéficié de soutien financier constant pour la réalisation de ses travaux. Le financement des interventions a aussi pu bénéficier de soutien ponctuel et à moyen terme, mais la stabilité de ce financement demeure toujours un défi. Dans les trois recherches-actions présentées, un certain financement a permis aux communautés, entreprises et organismes de développer certains services. Lors de la première expérience, ces services ont dû se déployer avec un financement minimal qui s'est accru au fil des ans. D'ailleurs, les deux principaux partenaires communautaires de ce projet reçoivent toujours un financement pour les actions qu'ils mènent auprès des pères. La seconde $\mathrm{a}$ pu bénéficier d'un financement qui est venu appuyer les activités développées par les entreprises tout au long de la recherche-action. Toutefois, l'arrêt du financement a empêché la poursuite des interventions de façon régulière. Enfin, les organismes participant à la troisième recherche-action ont pu embaucher pour une durée limitée les intervenants de milieu grâce au soutien d'organismes gouvernementaux ou philanthropiques. Cette situation illustre à quel point le financement d'interventions pour les pères est souvent considéré comme exceptionnel ou relevant de démarches exploratoires. Pourtant, ces actions devraient être considérées 
comme étant valables, au même titre que celles qui appuient les mères ou la famille et comme parties intégrantes des organismes familles et des services de santé et sociaux. De plus, les intervenants doivent prendre beaucoup de temps pour solliciter plusieurs partenaires afin d'espérer réaliser leur travail de façon efficace grâce à un appui financier. Sans quoi, la promotion de l'engagement paternel ne pourra se réaliser concrètement.

\section{Une passerelle entre les savoirs et les services destinés aux pères}

"Les connaissances accumulées durant ces années d'activités de l'équipe ProsPère reposent sur les échanges avec des pères et des mères vivant toutes sortes de situations, mais ayant en commun le désir de participer au mieux-être de leurs enfants. Elles se sont aussi construites sur les savoirs pratiques de tous ces intervenants rencontrés au fil des ans. »
Les connaissances accumulées durant ces années d'activités de l'équipe ProsPère reposent sur les échanges avec des pères et des mères vivant toutes sortes de situations, mais ayant en commun le désir de participer au mieux-être de leurs enfants. Elles se sont aussi construites sur les savoirs pratiques de tous ces intervenants rencontrés au fil des ans. Cette passerelle entre les savoirs théorique et pratique permet de mieux cerner les besoins des pères et d'identifier les conditions nécessaires à la réussite des pratiques auprès d'eux. Ce type de collaboration permet également de documenter les réalités vécues par les intervenants sur le terrain. De plus, cette association chercheurs-intervenants permet le transfert de ces connaissances auprès des nombreux partenaires associés à l'élaboration, la réalisation et l'évaluation de ces projets. Elle souligne finalement l'importance pour les chercheurs de s'associer aux terrains, non seulement pour avoir accès aux données qui les intéressent, mais aussi pour acquérir de nouvelles connaissances, lesquelles reflèteraient celles que possèdent déjà les intervenants qui oeuvrent dans ce champ de pratique.

De plus, ce dialogue entre chercheurs et intervenants a comme résultat la mise en place d'actions et de services pour les pères. Sans être des recettes miracles pour promouvoir l'engagement des pères à l'égard de leurs enfants, elles méritent d'être considérées pour ce qu'elles sont : des efforts collectifs pour appuyer les 
pères et accroître leur pouvoir d'agir en misant sur leurs forces et en suscitant, en renforçant et en maintenant l'engagement qu'ils ont face à leur enfant. Enfin, ce constant va-et-vient entre les connaissances théoriques et pratiques permet de poursuivre une réflexion sur l'évolution du rôle du père et les façons dont nous souhaitons aider les pères. Ce partage permet de briser les archétypes limitant les pères à certains aspects de la vie de famille et de leur enfant et de favoriser la création de nouveaux modèles de pères et de mesures pour les promouvoir. Il ne nous reste qu'à espérer que les autorités politiques et les différents réseaux concernés par l'avenir des enfants appuient concrètement ces pratiques et leur permettent de poursuivre ce travail.

\section{Gilles Forget,}

Conseiller en promotion de la santé, Direction de santé publique de l'Agence de la santé et des services sociaux de Montréal

1301, rue Sherbrooke est

Montréal (Québec) H1L 1M3

gforget@santepub-mtl.qc.ca

gillesforget@hotmail.com

514-524-2400, \#3404

Télécopieur : 514-528-2426

\section{Bibliographie}

AINSWORTH, Mary D.S. (1972). «Attachment and dependency :A comparison », Sous la direction de J.L. Gewirtz, Attachment and dependency, Washington, D.C., V.H.Winston.

ALLEN, Sarah, et Kerry DALY (2002). "The effects of father involvement : A summary of the research evidence " The FII-ONews, Vol.1, p. 1-11.

ARAMA, Dominique, et Camil BOUCHARD (1996). Recension des projets d'intervention ayant trait à la paternité dans la grande région de Montréal, Cahiers du G.R.A.V.E., Montréal, UQAM, GraveArdec, Vol.3, No1.

ARAMA, Dominique (1997). Promotion du rôle des pères : Inventaire des ressources et projets d'intervention spécifiques à la paternité au Québec, Québec, ministère de la Santé et des Services sociaux.

BELSKY, Jay (1984). "The determinants of parenting : A process model ", Child development, Vol.55, p. 83-96.

BLANKENHORN, David (1995). Fatherless America : Confronting our most urgent social problem, NewYork, Basic Books. 
BOLTÉ, Christine, et collab. (2002). Sur le terrain des pères. Projets de soutien et de valorisation de la paternité, Montréal, UQAM, Grave-Ardec.

BOWLBY, John (1969), Attachment and loss, New-York, Basic Books.

BRONFENBRENNER, Urie (1979). The ecology of human development, Cambridge MA, Harvard University Press Bulletin.

DEVAULT, Annie, et collab. (2005). Les caractéristiques des trajectoires de vie comme facteurs sous-jacents à l'engagement paternel. Le cas des jeunes ayant complété le programme d'une entreprise d'insertion, Québec, Fonds québécois de la recherche sur la société et la culture.

DUBEAU, Diane (2007). «Un partage des connaissances sur le transfert des connaissances, ProsPère », $3^{e}$ Séminaire Grave-Ardec, Montréal, Canada.

DOHERTY, William J., et Edward F. KONESKI et Martha F. ERICKSON (1998). « Responsible fathering : An overview and conceptual framework ", Journal of Marriage and family, Vol.60, p. 277-292.

FOR GET, Gilles, et collab. (2008). « Relais-Pères, working with vulnerable fathers », Father Involvement Research Alliance Conference, Toronto, Canada.

FOR GET, Gilles, et Dominic BIZOT (2006). Pères en mouvement, pratiques en changement, édition internationale. Guide du formateur et guides des participants ( $\left.N^{o} 1-6\right)$, Montréal, Direction de santé publique de l'Agence de la santé et des services sociaux de Montréal.

Forget, Gilles, et collab. (2005). "Les services destinés aux pères, une description et un regard sur l'évolution des pratiques canadiennes ", Enfances, familles, générations. $\mathrm{N}^{\circ} 3$, http:/www.uqtr.ca/efg.

FOR GET, Gilles, et Francine OUELLET (2002). Involving fathers, Evolving Practices, Guide du formateur et guides des participants ( $\left.N^{o} 1-4\right)$, Montréal, Direction de santé publique de l'Agence de la santé et des services sociaux de Montréal.

GAGNON, Marie-Noëlle, et Daniel PAQUETTE (2009). " La co-parentalité dans le système familial ", sous la direction de Dubeau, Diane, Devault, Annie et Gilles Forget, La paternité au $21^{e}$ siècle, P.U.L., Québec, Canada.

GOODMAN, Robert M., Frank C.WHELLER et Paul R. LEE (1995). «Evaluation of the heart to heart project : Lessons from a community-based chronic disease prevention project ", American Journal of Health Promotion, Vol.9 No 6, p. 443-445.

GOUVERNEMENT DU QUÉBEC (2008). Politique de périnatalité, 2008-2018, Québec, ministère de la Santé et Services sociaux du Québec.

HAWKINS, Allan J., et David C. DOLLAHITE (1997). Generative Fathering: Beyond deficit perspectives, Thousand Oaks, CA, Sage Publications.

HOBSON, Barbara, et David MORGAN, (2002). "Introduction ", sous la direction de Barbara Hobson, Making Men into Fathers : Men, Masculinities and the Social Politics of Fatherhood, NewYork, Cambridge University Press.

LAMB, Michael E., et collab. (1987). «A biosocial perspective on paternal behaviour and involvement ", sous la direction de J. B. Lancater, J. Altman, \& A. Rossi, Parenting across the lifespan : Biosocial perspectives, New York, Academic Press.

LAMB, Michael.E., Joseph H. PLECK et James A. LEVINE (1985). «The role of the father in child development : The effects of increased paternal involvement ", sous la direction de B.B. Lahey et A.E. Kazdin, Advance in Clinical Child Psychology, New York, Plenum. 
LE CAMUS, Jean (2004). Le vrai rôle du père. Paris, Odile Jacob.

MAR GOLIN, Gayla, Elana B GORDIS et Richard S.JOHN (2001). "Coparenting :A link between marital conflict and parenting in two-parent families ", Journal of Family Psychology, Vol.15, N¹, p. 3-21.

OUELLET, Francine, Marie-Pierre MILCENT et Annie DEVAULT (2006). "Jeunes pères vulnérables :Trajectoires de vie et paternité ", Nouvelles pratiques sociales, $\mathrm{N}^{\circ} 18$, p. 156-171.

OUELLET, Francine, et Gilles FORGET (automne 2003). "Pères en mouvement, pratiques en changement : une formation pour favoriser le transfert des connaissances ", Reflets, Revue ontaroise d'intervention sociale et communautaire, Vol.9, Nº 2, p. 222-240.

OUELLET, Francine, et Gilles FOR GET (2001). Pères en mouvement, pratiques en changement, Guide du formateur et guides des participants $\left(N^{o} 1-4\right)$, Montréal, Direction de santé publique de l'Agence de la santé et des services sociaux de Montréal.

PAQUETTE, Daniel (2004a). "Theorizing the father-child relationship : Mechanisms and developmental outcomes ", Human Development, Vol.47, N 4, p. 193-219.

PAQUETTE, Daniel (2004b). " La relation père-enfant et l'ouverture au monde ", Enfance, Vol.2, p. 205-225.

PITT, Edward W. (2001). "New Expectations : Community strategies for responsible fatherhood", Présences de pères : Actes du premier symposium national sur la place et le rôle du père, Montréal, Direction de santé publique de la Régie régionale de la santé et des services sociaux de Montréal-Centre.

PLECK, Joseph H., et Bernard P.MASCIADRELLI (2004). « Paternal involvement by U.S. Residential fathers. Levels, Sources, and Consequences ", sous la direction de Michael E. Lamb, The role of the father in child development (4th ed.), New Jersey, John Wiley \& Sons, Inc.

RISSEL, Charles, et Neil BRACHT (1999). "Assessing community needs, resources, and readiness : Building on strengths ", sous la direction de Neil Bracht, Health promotion at the Community Level: New Advances, Sage Publications, Thousand Oaks.

TURCOTTE, Geneviève, et collab. (2008). "Relais-Père : un projet de recherche-action pour rejoindre et soutenir les pères en situation d'exclusion ", Congrès de l'Association des Centres Jeunesse du Québec, Montréal, Canada.

TURCOTTE, Geneviève, et Gilles FORGET (2008). «La présentation des résultats du projet de recherche-action Relais-Pères ", 3 SU-PÈRE Conférence, Montréal, Canada.

TURCOTTE, Geneviève, et collab. (2008). «Le projet Relais-Pères Une approche novatrice pour favoriser l'engagement paternel et l'insertion sociale de pères vulnérables ", Congrès de l'Association des Centres jeunesse du Québec, Montréal, Canada.

TURCOTTE, Geneviève, Nicole DESJARDINS et Francine OUELLET (2001). À Pointe-Calumet, on fait place aux pères : analyse d'implantation d'Initiative Place-O-Pères, un projet d'action communautaire sur l'engagement paternel Montréal : Régie régionale de la santé et des services sociaux de MontréalCentre et Direction de la santé publique de Montréal-Centre.

VENET, Christine (2006). Effet de la formation Pères en mouvement, pratiques en changement sur les participants et les organismes, Montréal, Centre de formation populaire. 


\section{Notes}

1 Conseiller en promotion de la santé, Agence de la santé et des services sociaux de Montréal, Direction de santé publique, aussi membre du Grave-Ardec et de F.I.R.A.

2 http://www.graveardec.uqam.ca

3 Bouchard Camil, (1994), Demande de subvention de fonctionnement d'équipe, Groupe de recherche sur la victimisation des enfants, Conseil québécois de recherche en santé.

4 Dans le premier site, un chercheur américain, Michael Lamb est venu rencontrer les partenaires et a assisté à une soirée d'improvisation sur la paternité. Dans l'autre site, des conférences sont intégrées au programme de la Semaine de la famille.

5 Pour plus d'informations sur ces deux dispositifs, voir le chapitre sur le transfert des connaissances Dubeau, D., A. Devault et G. Forget (éd.) (2009). «La paternité au $21^{\text {e }}$ siècle », P.U.L.

6 La présentation des résultats s'est faite en novembre 2008 au colloque du Father Involvement Research Alliance (F.I.R.A.) à Toronto, au Colloque, au Congrès de l'Association des Centres jeunesse du Québec et à la troisième SU-PÈRE Conférence à Montréal. 